\title{
Ten Years Trend Analysis of Malaria Prevalence and its Correlation with Climatic Variables in Sibu Sire District, East Wollega Zone, Oromia Regional state, Western Ethiopia: A Retrospective Study
}

\author{
Temesgen Gemechu ${ }^{1}$, Abdi Samuel ${ }^{2^{\star}}$ and Delensaw Yewhalaw ${ }^{1}$ \\ ${ }^{1}$ Department of Biology, College of Natural Sciences, Jimma University, PO Box: 378, Jimma, Ethiopia \\ ${ }^{2}$ Department of Medical Laboratory Sciences, College of Medical and Health Sciences, Wollega University, \\ PO Box: 395, Nekemte, Ethiopia
}

\begin{tabular}{|c|c|}
\hline Abstract & Article Information \\
\hline $\begin{array}{l}\text { Malaria is one of the most devastating diseases in the World and caused by a protozoan } \\
\text { parasite of the genus Plasmodium. The disease remains one of the most important causes } \\
\text { of human morbidity and mortality with enormous medical, economic and emotional impact } \\
\text { in the world, and in most African countries including Ethiopia. The complexity of the } \\
\text { disease control process, expensiveness of the control program, resistance of the parasite } \\
\text { to anti-malarial drugs and vectors to insecticides are some of the challenges. The aim of } \\
\text { the study was to assess the ten years trend analysis of malaria prevalence and its } \\
\text { association with climatic variables in the Sibu Sire district, Western Ethiopia. Ten years } \\
\text { (2004-2013) malaria clinical and epidemiological data were collected from health facilities } \\
\text { and climatic variables data from Ethiopian Meteorological agency. The data were analyzed } \\
\text { using SPSS software package } 16.0 \text {. Pearson's correlation analysis was conducted to see } \\
\text { the correlation between plasmodium species and climatic variables. Within the last decade } \\
\text { (2004-2013) a total of } 30,070 \text { blood films were examined for malaria in Sire health center } \\
\text { and of this } 6036 \text { (20.07\%) microscopically confirmed malaria cases were reported in the } \\
\text { health center and } P \text {. falciparum becoming a predominant species. The result showed that } \\
\text { maximum temperature, mean temperature and average relative humidity showed } \\
\text { significant association with malaria }(P<0.01) \text {. But minimum temperature ( } P=0.094) \text { and } \\
\text { rainfall ( } P=0.729) \text {, were not significant. In addition, regression analysis suggested that } \\
\text { minimum temperature, rainfall, and average relative humidity }(P<0.001) \text { were statistically } \\
\text { significant but the mean temperature }(P=0.706) \text { was insignificant. In conclusion the trend of } \\
\text { malaria in the study area had a reducing but a fluctuating pattern and some of the } \\
\text { metrological variables such as minimum temperature, rainfall, and average relative } \\
\text { humidity were statistically significant. } \\
\text { Copyright@2015 STAR Journal, Wollega University. All Rights Reserved. }\end{array}$ & $\begin{array}{l}\text { Article History: } \\
\text { Received : 28-09-2015 } \\
\text { Revised : 19-12-2015 } \\
\text { Accepted : 24-12-2015 } \\
\text { Keywords: } \\
\text { P. falciparum } \\
\text { Malaria } \\
\text { Morbidity and mortality } \\
\text { Climatic Variables } \\
\text { Economic impact } \\
\text { Epidemiological data } \\
{ }^{*} \text { Corresponding Author: } \\
\text { Abdi Samuel }\end{array}$ \\
\hline
\end{tabular}

\section{INTRODUCTION}

Malaria is one of the most devastating diseases in the World. There are ninety seven countries and territories with ongoing malaria transmission, and seven countries in the prevention of reintroduction phase, making a total of 104 countries and territories in which malaria is presently considered endemic. Globally, an estimated 3.4 billion people are at risk of malaria. WHO estimates that 207 million cases of malaria occurred globally in 2012 (WHO, 2013). The disease remains one of the most important causes of human morbidity and mortality with enormous medical, economic and emotional impact in the world (WHO, 2011), with2-3million deaths occurring each year (Snow et al., 2005). It is the leading cause of death in children under the age of 5 years and pregnant women in developing countries (Martens, 2000; Lagerberg, 2008).

It is estimated that $68 \%$ of the population lives in malarious areas and three quarters of the total land mass is regarded as malarious $(\mathrm{MoH}, 2008)$. $P$. falciparum and $P$.vivax are the two predominant malaria parasites, distributed all over the country and accounting for $60 \%$ and $40 \%$ of malaria cases, respectively $(\mathrm{MoH}, 2002)$.

Malaria transmission in Ethiopia is seasonal and unstable, depending mostly on altitude and rainfall (Deressa et al., 2003). The two main seasons for transmission of malaria in Ethiopia are September to November, sometimes extended to December after heavy summer rains, and March to May, after the light rainy shower. Malaria epidemics are relatively frequent involving highland or highland fringe areas, mainly areas 1,000-2,000 meters above sea level, in which the population lacks immunity to malaria (Endeshaw et al., 2008). Meteorological factors have been considered as important drivers of malaria transmission by affecting both malaria parasites and vectors directly or indirectly 
Temesgen Gemechu et alo,

(Pemola and Jauhari, 2013). Besides this, other factors, such as any change in land use patterns and construction of water control could have considerable effects on malaria transmission (FMOH, 2007).

Changes in temperature, rainfall, and relative humidity due to climate change are expected to influence malaria directly by modifying the behavior and geographical distribution of malaria vectors and by changing the length of the life cycle of the parasite. Climate change is also expected to affect malaria indirectly by changing ecological relationships that are important to the organisms involved in malaria transmission (the vector, parasite, and host) (Abebe et al., 2011). Because of those challenges malaria is one of the main public health problems in the study area. Therefore, this study is initiated to analysis the ten years trend of malaria prevalence and to determine any patterns of correlation existing between meteorological factors and malaria over the last decade in the study area.

\section{MATERIALS AND METHODS}

\section{Study Area and Period}

The study was conducted in Sibu Sire district, East Wollega Zone of Oromia Regional State, Western Ethiopia from 2004-2013. It is one of the districts in east Wollega Zone and is located $281 \mathrm{Km}$ in West from Addis Ababa and $50 \mathrm{~km}$ East from Nekemte, the administration town of East Wollega Zone. This district is bordered in the East by Gobu Seyo, in the West by WayuTuka, in South by Wama Hagalo and Billo Boshe and on the North by Gudeya Bila and Guto Gida. This district has 22 kebeles from these 19 kebeles are rural and 3 municipals. The study area has a total population of 124,304 and from these urban dwellers male6744 and female 6954, rural dwellers male 54,920 female 55,686 .Sibu sire district has an estimated population density of 27.8 people per square kilometer (SSDA, 2013).

\section{Topography and Climate}

The altitude of the district ranges between 1360masl to 2500 masl.There is three agro-ecological zones represented in this district. The majority $(74.3 \%)$ of the district is classified as mid-land with lowland (18.27\%) and only $7.53 \%$ is considered as highland. The minimum, maximum and mean temperature of this area was 14.09 ${ }^{\circ} \mathrm{C}, 27.30{ }^{\circ} \mathrm{C}$, and $22.55{ }^{\circ} \mathrm{C}$, respectively. The highest temperature occurs in February and March. The lowest temperature occurs in July and august. The annual average rainfall of the district is $1295 \mathrm{~mm}$ (RLEPOSSD, 2013).

\section{Study Design and Population}

A retrospective study was employed to collect malaria epidemiological data from district health services to determine the ten year trend prevalence of malaria by reviewing blood film malaria record from Sire health center and metrological variables were collected from National Meteorology Agency.

In the study area peripheral smear examination of Giemsa stained blood film is used as the gold standard in confirming the presence of the malaria parasite as per the WHO protocol. The study participants were all individuals diagnosed for malaria using giemsa stained preparation and the data registered on laboratory registration book during the study period (2004 -2013).
Sci. Technol. Arts Res. J., Oct-Dec 2015, 4(4): 99-105

\section{Data Processing and Analysis}

The data were analyzed using SPSS version 16.0 software package. Descriptive statistics was employed to present overall malaria prevalence, age and sex specific malaria, correlation with climatic variables. Average yearly mean temperature, total rainfall and relative humidity (January through December for each year) were calculated. All data from meteorological and clinical records were checked for completeness and cleaned of any inconsistencies. To observe the correlation between meteorological variables and malaria cases, the monthly malaria cases were regarded as the dependent variables, while meteorological variables such as monthly maximum, minimum and mean temperature, total monthly rainfall and monthly relative humidity were independent variables. Pearson's correlation analysis was conducted to examine the type and strength of relationship between meteorological variables and malaria. Then to observe independent effect of each independent variable on outcome, variable linear regression was fitted. Since there might be auto-correlation among independent variables over time, autocorrelation analysis was conducted.

\section{Ethical Considerations}

Ethical clearance was obtained from Jimma University College of Natural Science and written consent was sought from the head of Sire district health office to undertake the study. Individual information was kept confidential and the names of individuals were removed from the data taken from health center and identified only by identification numbers and the results were communicated in an aggregated manner.

\section{RESULTS}

During the study period (2004-2013) a total of 30,070 blood films were requested for malaria diagnosis in Sire health center and 6036 (20.07\%) microscopically confirmed malaria cases were reported in the district with mean malaria cases of 603.6. A minimum (1.6\%)number of microscopically confirmed malaria cases being reported in 2008 and the maximum $(31.2 \%)$ microscopically confirmed cases of malaria being reported in 2004. The prevalence of malaria varies between sex and among place of residence, age ,month, season and year. In addition, the types of plasmodium species and the drug used within these years also varied.

The result of the study showed that malaria prevalence was higher in males than females. From the infected patients $(53.6 \%)$ were male and $(46.4 \%)$ were female but the difference was not statistically significant $(p=0.998)$ According to the data review in the last ten years in the study area for the Prevalence of malaria parasites in relation to residence area in Sibu Sire district people who live in rural areas were more affected by malaria than those who live in urban areas. The infection rates among rural people were $(69.3 \%)$ and urban people were $(30.7 \%)$. There was no significant difference $(p=0.436)$ between rural and urban residences.

Malaria case was reported in all age groups in the area but the age group between 15-44 years was more affected, with a prevalence rate of $(48.1 \%)$, followed by 5 14 years old and $1-4$ years old with the prevalence rate $(28.2 \%)$ and $(15.4 \%)$, respectively. On the other hand, children below 1years old and above 64 years old were less affected with prevalence rate of $2.3 \%$ and $1 \%$, 
Temesgen Gemechu et alo,

respectively. But the difference was statistically not significant $(p=0.712)$.

Malaria was reported in all years in the study area but in 2004 , the prevalence rate was high $(31.2 \%)$, followed by 2010,2005 with the prevalence rate $(13.7 \%)$ and $(13 \%)$, respectively. Similarly, the prevalence rate of malaria in 2012, 2013 and 2009 was (9.8\%), (9.7\%) and $(9.3 \%)$, respectively. But the rest years less number of

Sci. Technol. Arts Res. J., Oct-Dec 2015, 4(4): 99-105

malaria prevalence was reported. When we observe figure 1 the $P$. falciparum highly decreased from2004 up to 2008 and again rise starting2009 in 2010 remarkably increase and in 2011 become below the prevalence of $P$.vivax. In contrast to these in $2011 P$. vivax prevalence was elevated a little above $P$. falciparum in the study area. The prevalence of mixed infection $(P$. falciparum and $P$. vivax) remained almost the same from 2004 up to 2013(figure 1).

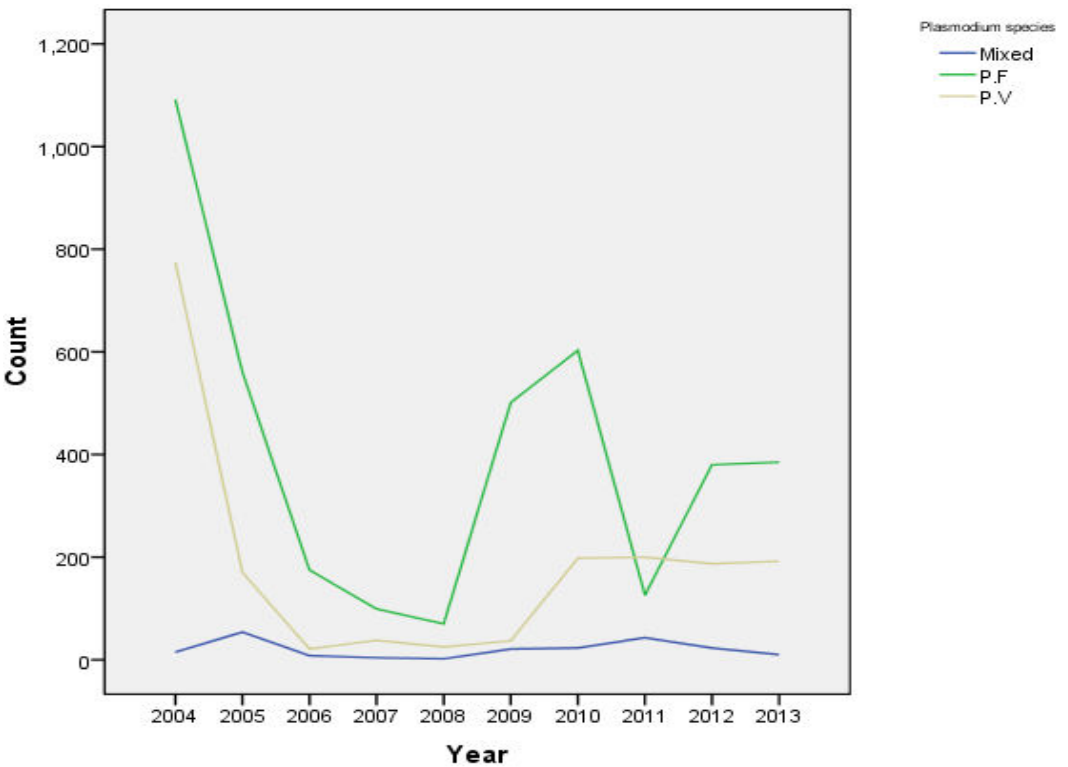

Figure 1: Trend of malaria prevalence with Plasmodium species and year in Sire health center, 2004 - 2013.

Despite the apparent fluctuation of malaria trends in the study area; malaria cases occurred in almost every season of the years. The highest peak of malaria cases in almost all years was observed during the wet season, with prevalence rate of $(68.36 \%)$ and the dry season with prevalence rate of $(31.64 \%)$, which was statistically significant $(P=0.001)$.

Malaria occurred in all months of the year with different fluctuation rate. The highest peak is in June with prevalence rate $18.9 \%$, followed by May, November, and July with prevalence rate $13.3 \%, 13.2 \%$, and $11.2 \%$, respectively. The prevalence rate in October, August, and September were $9.4 \%, 8.7 \%$, and $7 \%$, respectively. On other hand, the spread of malaria in December, January,
February and March were low when compared with other months with prevalence rate of $4.2 \%, 4.2 \%, 4 \%$ and $2.9 \%$ respectively. Although a seemingly uniform monthly health center record, having higher values were recorded in Sibu Sire district starting from May up to November.

Figure 2 below showed that there was fluctuated trend of P.falciparum and P.vivax throughout the year in different months. The peak malaria cases were seen in June, May, and November months with high P.falciparum and then $P$.vivax respectively. In contrast, the less malaria cases were confirmed in March, April, February, December, and January months respectively. Almost the mixed cases less fluctuate through each month.

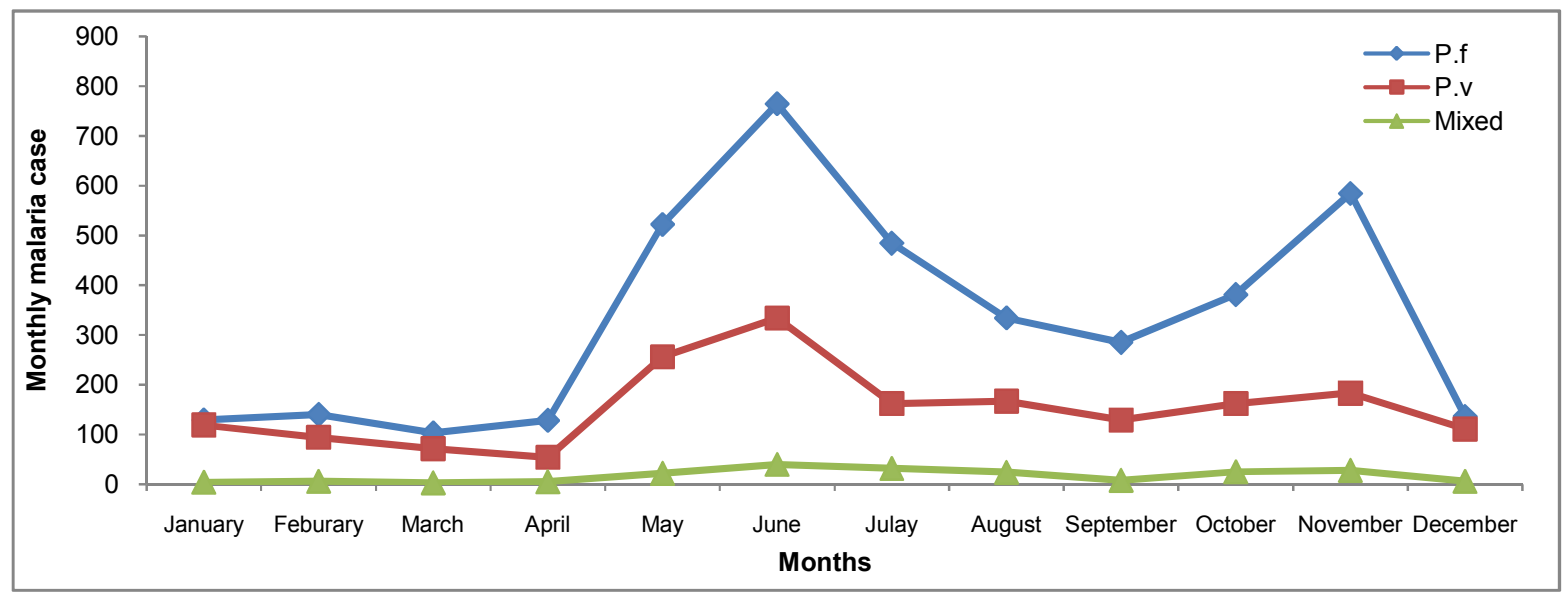

Figure 2: Trend of malaria prevalence with Plasmodium species and month in Sire health center, $2004-2013$ 


\section{Temesgen Gemechu et al.,}

Regarding the identified plasmodium species, both species of plasmodium were reported in each year with $P$. falciparium being the predominant species in the study area. P. vivax accounted for $66.1 \%$ and $30.5 \%$ of malaria cases, respectively and the mixed (both P.falciparum and $P$.vivax) accounted $3.4 \%$. In the year $2011 P$. falciparum was decreasing while $P$. vivax was increasing, which showed that there was a trend shift from $P$. falciparum to
Sci. Technol. Arts Res. J., Oct-Dec 2015, 4(4): 99-105

$P$. vivax in the study area.

Of the cases diagnosed for malaria from different kebeles the highest $(23.7 \%)$ malaria confirmed cases were from Jarso Wama kebeles, followed by Sire-02 kebele(16.9\%), Sire-01 kebele(13.9\%\%), Bikila kebele(13.2\%) and Lalisa(9.4\%). Figure 3 below showed the fluctuating trends of malaria in the study area.

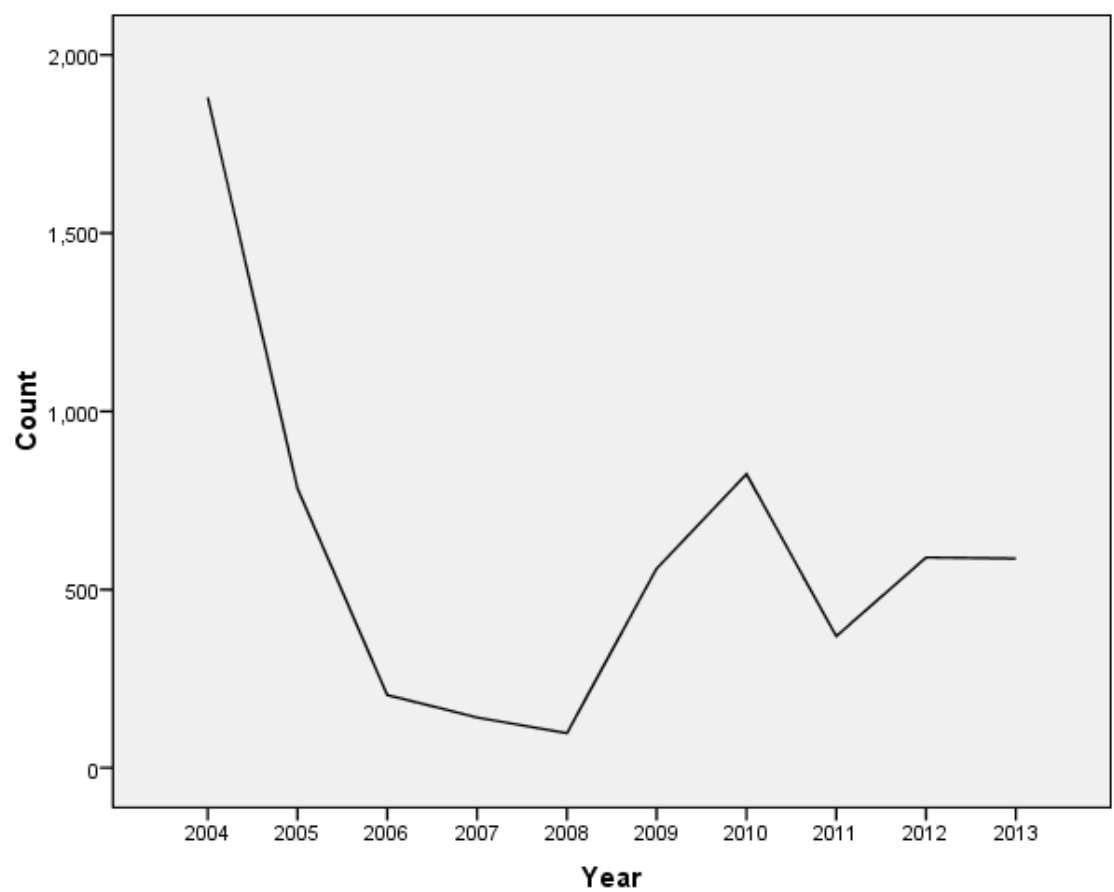

Figure 3: Annual trends in total malaria cases in Sire Health Center, $2004-2013$

\section{Correlation between Malaria and Meteorological Variable}

The study area is generally characterized by moderate climate with a mean annual maximum and minimum temperature $27.3^{\circ} \mathrm{C}$ and $14.09^{\circ} \mathrm{C}$. The annual rainfall ranged from $650 \mathrm{~mm}$ to $2590 \mathrm{~mm}$. The annual mean relative humidity $68.66 \%$ from 2004 to 2013 in study area.

In general, the relationship between malaria cases and meteorological variables were checked by using mean and standard deviation and also further checked by Pearson's correlation and linear regression analyses (table 1).

Depending on climate variable in 2004 the mean minimum temperature higher than the mean minimum temperature in2008 and also average relative humidity of 2004 was greater than the average relative humidity in 2008. Moreover, the annual mean temperature of the study area from 2004 to 2013 was showed that low temperature $\left(18.67^{\circ} \mathrm{C}\right)$ in 2011 and high temperature $\left(26.87^{\circ} \mathrm{C}\right)$ in 2004 . A high fluctuating trend in temperature was observed during the study period. The condition of rain fall in the study area in the past ten years (20042013 ) indicated that minimum rainfall $650 \mathrm{~mm}$, maximum rain fall $2590 \mathrm{~mm}$ and mean annual rainfall $490 \mathrm{~mm}$. However, a high fluctuating trend of rainfall was recorded through the years of 2004 to 2013.

Pearson's correlation analysis was employed to assess the correlation between malaria and climatic variables (temperature, rainfall, and relative humidity). It showed that maximum temperature $(P=0.007)$, mean temperature $(P=0.001)$ and average relative humidity $(P=0.001)$ showed significant correlation with malaria. But annual mean minimum temperature $(P=0.094)$ and annual rainfall $(P=0.729)$ were not statistically significant.

Table 1: Mean and Standard deviation of different species of Plasmodium with climate variable

\begin{tabular}{|c|c|c|c|c|c|}
\hline $\begin{array}{l}\text { Plasmodium } \\
\text { species }\end{array}$ & $\begin{array}{l}\text { Mean temp. } \\
\text { Mean( }( \pm S D)\end{array}$ & $\begin{array}{l}\text { Minimum temp. } \\
\text { Mean(士SD) }\end{array}$ & $\begin{array}{l}\text { Maximum temp. } \\
\text { Mean(士SD) }\end{array}$ & $\begin{array}{c}\text { Rain fall } \\
\text { Mean( }( \pm S D)\end{array}$ & $\begin{array}{l}\text { Relative humidity } \\
\text { Mean(土SD) }\end{array}$ \\
\hline Mixed & $19.98 \pm 3.82$ & $13.7 \pm 3.4$ & $25.38 \pm 5.54$ & $7.42 \pm 7.28$ & $67.7 \pm 15.78$ \\
\hline P.F & $22.33 \pm 3.26$ & $14.08 \pm 1.38$ & $27.45 \pm 2.88$ & $4.79 \pm 3.93$ & $69.39 \pm 16.03$ \\
\hline P.V & $23.35 \pm 4.35$ & $14.19 \pm 2.33$ & $27.19 \pm 3.86$ & $4.84 \pm 5.06$ & $67.24 \pm 16.59$ \\
\hline Total & $22.54 \pm 3.69$ & $14.09 \pm 1.79$ & $27.3 \pm 3.34$ & $4.90 \pm 4.47$ & $68.66 \pm 16.22$ \\
\hline$P$-value & 0.001 & 0.004 & 0.001 & 0.001 & 0.001 \\
\hline
\end{tabular}


Temesgen Gemechu et alo,

The malaria cases were positively correlated $(+0.028)$ with annual minimum temperature, $(+0.128)$ with annual average mean temperature, and $(+0.005)$ annual total rainfall. The negative correlation of malaria cases were observed (-0.037) with annual average maximum temperature and $(-0.062)$ with annual average relative humidity.

\section{DISCUSSION}

The result of this study revealed that during the last ten years, a fluctuating trend of occurrence of malaria cases was observed in SibuSire district almost similar to the study conducted in Kola Diba health center, North Gonder by (Abebe et al., 2012) and also the study done in Butajira by(Tesfaye et al., 2012). An increase in malaria cases occurrence with peak cases occurring in 2004 was similar with the thesis conducted in Mvomero, by (Xiaochen, 2013), and malaria cases were reduced the following three consecutive years (2006-2008) but a remarkable increase in 2010 was observed. Except for the year 2011, the remarkable increment of total malaria cases was mainly due to an increase of $P$.falciparum with little increase of $P$.vivax. But for 2011 total malaria cases, P.vivax contributed more than P.falciparum the same to the study done by (Abebe et al., 2012) in Kola Diba health center and also Similarly the study conducted around Gilgel Gibe show that in 2008 and 2010 when the $P$. vivax prevalence was elevated a little above $P$. falciparum (Sena,2014). Resistance of P.vivax to the commonly used drug (chloroquine) during these years may have contributed to total malaria case occurrence (WHO, 2003; MoH, 2004; Jima, 2005).

A decrease in malaria cases occurrence after the 2004 maximum occurrence was observed. The increased attention to malaria control and preventive activities by different responsible bodies, increased awareness of the community on use of ITNs and other malaria control activities, increased accessibility of ITNs to community, increment of budget for malaria control and prevention activities might contributed the decrement in malaria case occurrence in addition to meteorological factors (Abebe et al., 2011; Abebe et al., 2012).

In this study, males were more affected than females. Because they stay outdoors to keep agricultural products at night when mosquito becomes active to find food (meal). The results were comparable with an earlier study, carried out by. (Jamaiah et al., 1998; Karunamoorthi and Bekele, 2009; Abebe et al., 2012; Getachew et al., 2013). The female population mostly stays back in the house, thus their contact with malaria infection is minimized. Malaria prevalence was more series in rural than urban because rural communities they do not use bed net appropriately. In addition their houses are full of cracks and holes so mosquito can easily enter it. The number of kebeles in the rural area is 19 and there is only 3 urban kebeles in the district. This has contributed to the increment of the number. This study had also showed similar result to the study done by (Abebe et al., 2011) in Jimma town, South-West Ethiopia.

The age group 15-44 years was more affected with malaria. This could be attributed to the fact that people in this age group stay outside till late evening for work as they are in productive age. This is consistent with a the study conducted by Abebe et al. (2012) in Kola Diba,
Sci. Technol. Arts Res. J., Oct-Dec 2015, 4(4): 99-105

North Gonder, and North West Ethiopia was obtained the same result.

Malaria occurred in almost every season of the year. The highest peak of malaria observed in summer. So the season before the dry season has rain and temperature that enough for Sporogonic cycles reproduction of Anopheles mosquitoes. A study by Jason (2007) in Democratic People's Republic of Korea has similar results in that the summer season has highest malaria incidence. The district have high irrigation farming in the Autumn Season these condition may be increase the number of malaria transmitting mosquitoes. The same result can be found in China and India Country (Peng et al., 2003; Pemola, 2006; Srinivasulu, 2013).

The study also showed that the highest peak of malaria infection was occurred in June with high occurrence of malaria which could be due to the fact that June is the starting time for Summer season and May the last month in autumn season was suitable condition for malaria disease increment that is the amount of temperature and rain enough for the reproduction of mosquitoes and Plasmodium species (Peng et al., 2003; Pemola, 2006).

$P$. falciparum was the predominant species followed by $P$. vivax in the study area and the prevalence rate accounted for 3991(66.1\%) and1842 (30.5\%) respectively. The study conducted in Sarbo, Kola Diba, Butajira health center and Metema Hospital by (Abebe et al., 2012; Karunamoorthi and Bekele, 2012; Solomon et al., 2012; Getachew et al., 2013) almost similar to these results. Other earlier studies have also documented the predominance of $P$. falciparum species in many places of Ethiopia.

The major climatic factors that have an impact on malaria transmission due to spatio-temporal changes in malaria vectors are temperature, relative humidity $(\mathrm{RH})$ and precipitation (Pampana, 1969). Increasing atmospheric temperature causes decrease in the duration of the gonotrophic cycle as well as the extrinsic incubation period, leading to increased biting frequency, and therefore, increased rate of malaria transmission (Molineaux, 1988).

Unlike some diseases, which flourish under one particular set of environmental conditions, the environmental factors that contribute to the transmission of malaria vary greatly from one ecological zone to another. Of all of these, the two most important ones are temperature and humidity. Organisms cease to develop in the mosquito when the temperature falls below $16^{\circ} \mathrm{C}$. At $20^{\circ}-30^{\circ} \mathrm{C}$, the parasites develop optimally in the vector. High humidity prolongs the life of the vector and transmission is extended under these conditions. In the human host, the parasite must function at $37^{\circ} \mathrm{C}$ or higher, since the infection induces a significant rise in core temperature during the height of the infection (Githeko, 2008; Anon, 2012).

Temperature rise is expected to increase transmission and prevalence of malaria by reducing the interval between mosquito blood meals, thus decreasing the time to produce new generations and by shortening the incubation period of the parasite in the mosquitoes. Sporogonic cycles take about 9 to 10 days at 
Temesgen Gemechu et alo,

temperatures of $28^{\circ} \mathrm{C}$ but higher than $30^{\circ} \mathrm{C}$ and below $16^{\circ} \mathrm{C}$ have negative impact on parasite development (Craig, 1999). Also the minimum temperature for $P$. falciparum and P.vivax parasite development approximates to $18^{\circ} \mathrm{C}$ and $15^{\circ} \mathrm{C}$, respectively and the daily survival of the vector is dependent on temperature as well. At temperatures between $16^{\circ} \mathrm{C}$ and $36^{\circ} \mathrm{C}$, the daily survival is about $90 \%$. The highest proportion of vectors surviving the incubation period is observed at temperatures between $28^{\circ}-32^{\circ} \mathrm{C}$. So, temperature of $20^{\circ} \mathrm{C}$ to $30^{\circ} \mathrm{C}$ and relative humidity greater than $60 \%$ are optimal for Anopheles survive long enough to acquire and transmit the parasite (Craig, 1999).

Rainfall plays an important role in malaria epidemiology because water not only provides the medium for the aquatic stages of the mosquito's life but also increases the relative humidity and thereby the longevity of the adult mosquitoes (McMichael and Martens, 1995). The impact of rainfall on the transmission of malaria is very complicated, varying with the circumstances of a particular geographic region and depending on the local habits of mosquitoes. Rains may prove beneficial to mosquito breeding if it is moderate, but may destroy breeding sites and flush out the mosquito larvae when it is excessive (McMichael and Martens, 1995).

The annual total rainfall was positively correlated that determines malaria transmission in the study area next to mean temperature and minimum temperature. Rainfall plays an important role in malaria epidemiology because water not only provides the medium for the aquatic stage of the mosquitoes' life cycle but also increases the relative humidity and then the longevity of the adult mosquitoes (McMichael and Martens, 1995).

Both the correlation and regression analyses suggests that temperature, rainfall and relative humidity act on yearly malaria case. Although all meteorological variables were less likely to predicts the occurrence of malaria in Sibu Sire district. This finding contradicts the findings in Dehradun, Uttaranchal, India (Anthony and McMichael, 2000), Shuchen County, China (Donald and Pend, 2000), Rwanda (Loevinsohn, 1994), Madagascar ( Bouma, 2003), and east Africa Highlands (Rogers, 2000) which concluded that meteorological variables were highly likely correlated with malaria occurrence and the prediction was higher than this finding with higher $\mathrm{R}$ square value. This variation might be due to the fact that this study was conducted in lowlands in which malaria is endemic. In lowlands, the factors that contribute to malaria transmission dynamics are microclimate variation due to anthropogenic effects and other non- climatic factors like, health system, population growth, population movement and others (Gebere-Mariam, 1984; Abeku et al., 2004).

In general, there was a fluctuation in malaria prevalence during the last ten years. Many factors might be responsible for seasonal changes, like climatic variables, ecologic and environmental factors, host and vector characteristics, and social and economic determinants such as change in health care infrastructure. Social, biological and economic factors such as mosquito control measures, population immunity, local ecological environment, governmental policy, availability of health facilitates and drug resistance also has an impact on malaria prevalence. Although there were different malaria
Sci. Technol. Arts Res. J., Oct-Dec 2015, 4(4): 99-105

control activities in each year, such as indoor residual spraying, environmental management, health education about malaria, distribution of LLINs and anti-malarial drugs and other activities to decrease mortality and morbidity of malaria, the prevalence is still sustained.

\section{CONCLUSIONS}

The study showed a decrease but fluctuating trend in the last ten years $(2004-2013)$. When compared with the previous prevalence of malaria currently there is a big difference that means malaria prevalence is highly reducing. Metrological variables such as maximum temperature mean temperature and average relative humidity showed significant correlation with malaria transmission. But malaria is still a major public health problem and deadly that needs sustainable intervention on variables such as keeping the ecology.

\section{Conflict of Interest}

Conflict of Interest none declared.

\section{REFERENCES}

Abebe, A., Dagnachew, M., Mikrie, M., Meaza, A., Melkamu, G. (2012).Ten year trend analysis of malaria prevalence in Kola Diba, North Gondar, Northwest Ethiopia. Parasites \& Vectors 5:173.

Abebe, A., Gemeda A., Wondewossen T., Lemu G. (2011). Climatic variables and Malaria transmission dynamics in Jimma town, South West Ethiopia. Journal of Parasites and Vectors 4:30

Abebe, A., Wondewosen, Ts., Lemu, G., and Gemeda, A. (2011).Urban malaria and associated risk factors in Jimma town, south-west Ethiopia. Malaria Journal 10:173.

Abeku, T., Dexlas, S.J., Borsboom, G.J. (2004). Effects of meteorological factors on epidemic malaria in Ethiopia: a statistical modeling approach based on theoretical reasoning. Parasitology 128:85-96.

Anon,(2012).MedicalEcology.http://www.medicalecology.org/ diseases/malaria/malaria.htm .last accessed, august 05, 2012.

Anthony, J., McMichael, A. (2000).Implications of climate change on malaria in Karnataka, India. London School of Hygiene and Tropical Medicine 25:34-39.

Bouma, M.J. (2003). Methodological problems and amendments to demonstrate effects of temperature on the epidemiology of malaria. Transactions of the Royal Society of Tropical Medicine and Hygiene 97 (2): 133139.

Craig, M.H., Snow, R.W., Sueur, D. (1999). A climate-based distribution model of malaria transmission in sub-Saharan Africa. Parasitology Today 15:105-111.

Deressa, W., Ali, A., Enqusellassie, F. (2003). Self-treatment of malaria in rural communities, Butajira, Southern Ethiopia. Bulletin of the World Health Organization 81 (4): 261-268.

Donald, K., Pend, BI. (2000). Climate variability and malaria, Shuchen County in China. Public Health report118:65-70.

Endeshaw, T., Gebre, T., Ngondi, J. (2008). Evaluation of light microscopy and rapid diagnostic test for the detection of malaria under operational field conditions: a household survey in Ethiopia. Malaria Journal 7:118. 
Temesgen Gemechu et alo,

FMoH (2007). Africa malaria day 2007 celebrations in Ethiopia, Joint press Release for the commemoration of the Africa Malaria Day: 1-98pp.

Gebere-Mariam, N. (1984). Highlights of malaria situation in Ethiopia: National Health Development Network. Addis Ababa; 5-18 October.

Getachew, F., Abiyu, W., Alemtegna, G., Ali, A., Tarekegn, H., Yenus, A., Belay, T., Yitayih, W., Abebe A. (2013). Prevalence of Malaria from Blood Smears Examination: A Seven-Year Retrospective Study from Metema Hospital, Northwest Ethiopia. Malaria Research and Treatment 2013 (2013): 704-730. recession. Nature 465: 342-345.

Githeko, A.K. (2008). Climate Change and possible impacts on population.HIV Resurgent Infections and population change in Africa; Carael, M., J.R.; Eds.; springer: New York, NY, USA.67-77pp.

Jamaiah, I., Anuar, A.K., Najib, N.A., Zurainee, M.N. (1998). Imported malaria: a retrospective study in University Hospital, Kuala Lumpur, a ten-year experience. Medicine Journal of Malaysia 53(1): 6-9.

Jima, D., Tesfaye, G., Medhin, A., Kebede, A., Argaw, D., Babaniyi, O. (2005).Efficacy of sulfadoxinepyrimethamine for the treatment of uncomplicated falciparum malaria in Ethiopia. East Africa Medicine Journal 82:391-95.

Karunamoorthi, K., Bekele M. (2009). Prevalence of malaria from peripheral blood smears examination: a 1-year retrospective study from the Serbo Health Center, Kersa Woreda, Ethiopia. Journal of Infection and Public Health 2(4):171-176.

Karunamoorthi K., Bekele M. (2012). Changes in Malaria Indices in an Ethiopian Health Centre: A Five Year Retrospective Analysis. Journal of Health Scope 1(3):118126.

Lagerberg, R. (2008).Malaria in pregnancy: a literature review. Journal of Midwifery Women's Health 53:209-215.

Loevinsohn, M.E. (1994). Climate warming and increased malaria incidence in Rwanda. Lancet 343:714-18.

Martens, P., Hall, L. (2000).Malaria on the move: Human population movement and malaria transmission. Emerging Infectious Diseases 6: 28-45.

McMichael, A.J., Martens, W.J.M., (1995). The health impact of global climate changes: grasping with scenarios, predictive models and multiple uncertainties. EcoHealth 1: 23-33.

$\mathrm{MoH}$ (2002).Guideline for malaria vector control in Ethiopia: malaria and other vector- borne diseases prevention and control team Diseases prevention. Addis Ababa, Control Department. March 2002.

$\mathrm{MoH}(2002 / 03)$. Health and health related indicator. Planning and Programming Department, Ministry of Health. Addis Ababa, Ethiopia.

$\mathrm{MoH}$ (2000). Malaria control profile. Disease prevention and Control Department. Commercial Printing Enterprise. Addis Ababa, Ethiopia.

$\mathrm{MoH}$ (2006). National five- year strategic Plan for malaria prevention \& control in Ethiopia; 2006-2010. Addis Ababa, Ethiopia. April 2006.
Sci. Technol. Arts Res. J., Oct-Dec 2015, 4(4): 99-105

$\mathrm{MoH}$ (2004). Guideline for malaria epidemic prevention and control in Ethiopia.2nd edition. FMoH, Addis Ababa, Ethiopia: July 2004: 1-2pp.

$\mathrm{MoH}(2004 / 5)$. A Health and health related indicators. Planning and Programming Department, $\mathrm{FMOH}$, Addis Ababa, Ethiopia. December 2005.

$\mathrm{MOH}$ (2010).Malaria Coordination and reflects collaborative discussions with the national malaria control programs and partners in country. President's Malaria Initiative; Ethiopia FY10, USAID.5-7pp.

$\mathrm{MOH}(2012)$.President'sMalarialnitiative.Availableonline:http:// pmi.gov/countries/mops/fy13/ghana_mop_fy13.pdf (accessed on 1 September 2013).11-12pp.

Molineaux, L. (1988). The epidemiology of human malaria as an explanation of its distribution, including some implications for its control. Malaria, principles and practice of Malariology, Werndorfer, W. H., and I.Mc Gregor, eds. Edinburg: Churchill livingstone.913-998pp.

Pampana, E. (1969) .Textbook of malaria eradication.2nd ed. London: Oxford University Press.

Pemola, N. D., Jauhari, R.K.(2006).Climatic variables and malaria incidence in Dehradun, Uttaranchal, India. Journal Vector Borne Disease43:.21-28.

Peng, B., Shilu, T., Ken, D., Kevin, A., Jinfa, N. (2003). Climatic Variables and transmission of Malaria: A 12 year Data Analysis in Shuchen Country, China. Public Health Report 118:65-71.

RLEPOSSD(2013). Rural Land Environmental Protection of Sibu Sire District, East Wollega Zone Oromia, Ethiopia.

Rogers, D.J., Randolph, S.E. (2000). The global spread of malaria in a future, warmer world. Science 289:17631766.

Sena, L., Deressa, W., Ali, A. (2014). Analysis of trend of malaria prevalence in south-west Ethiopia: a retrospective comparative study. Malaria Journal 13:188

Snow, R.W., Guerra, C.A., Noor, A.M., Myint, H.Y., Hay, S.I.(2005).The global distribution of clinical episodes of $P$. falciparum malaria. Nature 434(7030):214-217.

Srinivasulu, N., Gujju, G., Reddya, N., Sambashiva, D. (2013). Influence of Climate Changeon Malaria Incidence in Mahaboobnagar district of Andhra Pradesh, India. International Journal of Current Microbiology and. Applied Science 2(5): 256-266

Tesfaye, S., Belyhun, Y., Teklu, T., Medhin, G., Mengesha, T., Petros, B. (2012). Malaria pattern observed in the highland fringe of Butajira, Southern Ethiopia: a ten-year retrospective analysis from parasitological and metrological data. Malaria World Journal 3: 5.

WHO (2011). Global Fund proposal development, WHO policy brief on malaria, Geneva, Switzerland.

WHO (2013). World Malaria Report2013. Avenue Appia, Geneva, Switzerland, ISBN 978924 156469, 4- 31pp.

Xiaochen, D. (2013). Malaria Trends and Diagnoses in Rural Health Facilities in Mvomero, Tanzania. MSc thesis, Duke University, UK. 